\title{
MINOR-ARC AND MAJOR-ARC GLOBAL SURFACE WAVE DIFFRACTION TOMOGRAPHY
}

Anatoli L. Levshin ${ }^{a, 1}$, Michael P. Barmin ${ }^{a}$, Michael H. Ritzwoller ${ }^{a}$, and Jeannot Trampert ${ }^{b}$

${ }^{a}$ Center for Imaging the Earth's Interior, Department of Physics, University of Colorado at Boulder, Campus Box 390, Boulder, CO 80309, USA.

${ }^{b}$ Department of Geophysics, University of Utrecht, P.O. Box 800213508 TA Utrecht, The Netherlands.

Keywords: Surface waves; Tomography; Phase velocity; Diffraction

\begin{abstract}
We discuss extending global surface wave diffraction tomography to accommodate major-arc dispersion measurements. The introduction of major-arc surface wave dispersion measurements improves path density and resolution in regions poorly covered by minor-arc measurements alone, as occurs in much of the southern hemisphere. The addition of major-arc measurements to the inversion for dispersion maps does not appreciably degrade the fit to the minor-arc measurements but significantly improves the fit to the major-arc measurements. For these reasons, we conclude that the addition of major-arc measurements is worthwhile in the interim until the broad-band network of ocean bottom or Antarctic stations is improved in the future.
\end{abstract}

\footnotetext{
${ }^{1}$ Corresponding author

E-mail address: levshin@ciei.colorado.edu
} 


\section{Introduction}

This paper extends current tomographic methods to invert measurements of surface wave dispersion for maps of the two-dimensional distribution of phase or group speeds regionally or over the globe. Barmin et al. (2001) previously described a method of surface wave tomography based on geometrical ray-theory with largely ad-hoc smoothing constraints. This method has been used in several studies of earth structure (e.g., Levshin et al., 2001; Ritzwoller et al., 2001; Shapiro et al., 2002). Ray-theory is a high frequency approximation, however, which is not justified in the presence of heterogeneities whose length-scale is comparable to the wavelength of the wave (e.g., Woodhouse, 1974; Wang \& Dahlen, 1995). For the ray approximation to be valid, the first Fresnel zone must be smaller than the scale-length of the heterogeneity, which places limitations on the lateral resolution of seismic models based on ray-theory. The Born or Rytov approximation for surface wave scattering (e.g., Woodhouse \& Girnius, 1982; Yomogida \& Aki, 1987; Snieder \& Romanowicz, 1988; Bostock \& Kennett, 1992; Friederich et al., 1993, Friederich 1999; Meier et al., 1997; Spetzler et al., 2001, 2002; Yoshizawa \& Kennett, 2002; Snieder, 2002) models the finite width of the surface wave sensitivity zone. Ritzwoller et al. (2002) discussed the use of this approximation in the context of global surface wave tomography, calling the resulting method global diffraction tomography. This method was the basis for a global three-dimensional (3-D) shear velocity model of the crust and upper mantle (e.g., Levin et al., 2002; Ritzwoller et al., 2003a, 2003b, 2004) based exclusively on minor-arc group and phase measurements. Some regions of the Earth, especially in the southern hemisphere, cannot be effectively covered by minor-arc paths due to the sparseness of seismic stations. The use of major-arc data for both the fundamental mode and overtone data (van Heijst et al., 1999) would significantly improve the spatial and azimuthal coverage particularly for studies of azimuthal anisotropy. Spetzler et al. (2002) discuss diffraction tomography for major-arc measurements, but minor and major-arc observations have been previously used in tomographic studies only under the assumption of ray-theory (e.g., Trampert \& Woodhouse, 2003).

In this paper we follow Spetzler et al. (2002) to extend diffraction tomography by redefining the zone of sensitivity and accommodating both minor-arc and major-arc measurements 
using the Born/Rytov approximation. We take the opportunity along the way to consider several variants of the sensitivity kernels for both major and minor-arc paths. Due to focusing effects at the antipodes of the source and the receiver, the structure of the major-arc surface wave sensitivity kernel is more complicated than for minor-arc measurements. We apply this approach to an update of the surface wave phase speed measurements obtained by Trampert \& Woodhouse $(1995,1996)$ and estimate the improvements in spatial resolution as well as the reliability of the resulting tomographic maps. We pay special attention to the southern hemisphere, and, particularly, to parts of the South Pacific and Antarctica where coverage by minor-arc paths remains much worse than in most of the northern hemisphere.

\section{Sensitivity Kernels for Minor and Major Arc Paths}

Under the Born/Rytov approximation, the perturbation to a surface wave travel time for source $i$ and receiver $j$ is written as an integral over the Earth's surface S:

$$
\delta t_{(n, q)}^{i j}(\nu)=\int_{S} K_{(n, q)}^{i j}(\mathbf{r}, \nu) v_{q}^{-1}(\mathbf{r}, \nu) m(\mathbf{r}, \nu) d S
$$

where

$$
m=\frac{\delta v_{q}(\mathbf{r}, \nu)}{v_{q}(\mathbf{r}, \nu)}
$$

$(n, q)$ is an ordered pair with $q$ designating the wave type (Rayleigh or Love) and $n$ specifying whether the measurement is for a minor-arc $(n=1)$ or a major-arc $(n=2)$ path, $\nu$ is the wave frequency, $\delta v_{q}(\mathbf{r}, \nu)$ is the perturbation to phase speed at location $\mathbf{r}$ relative to the reference model $v_{q}(\mathbf{r}, \nu)$, and $K_{(n, q)}^{i j}$ is the sensitivity kernel defined for the particular sourcereceiver configuration.

The shape of the sensitivity kernel depends both on frequency and epicentral distance. Following Spetzler et al. (2001, 2002), if epicentral distance $\Delta<\pi$ (a minor-arc path), then $K_{(n, q)}=K_{(1, q)}(\Delta, \theta, \phi, \nu):$

$$
K_{(1, q)}(\Delta, \theta, \phi, \nu)=\frac{\cos \theta}{2 \Delta \delta \nu} \int_{\nu_{0}-\delta \nu}^{\nu_{0}+\delta \nu} W(\nu) \sqrt{\frac{\nu R_{0} \sin \Delta}{H(\theta, \phi) v_{q}\left(\theta, \phi, \nu_{0}\right)}} \sin \left[\frac{\pi \nu R_{0} \theta^{2} \sin \Delta}{H(\theta, \phi) v_{q}\left(\theta, \phi, \nu_{0}\right)}+\frac{\pi}{4}\right] d \nu
$$


where $H(\Delta, \phi)=\sin \phi \sin (\Delta-\phi)$ and $R_{0}$ is the Earth's radius. For simplicity of presentation, we omit the source and receiver indices and use a coordinate system centered on the greatcircle linking the source and receiver $(\theta, \phi)$ and the assumption that the great-circle lies along the equator. In this way, $\phi$ is measured along the great-circle $(0<\phi<\Delta)$ and $\theta$ is measured in the transverse direction, along meridians from the equator $\left(-\frac{\pi}{2}<\theta<\frac{\pi}{2}\right)$. In practice, a measured travel time perturbation depends on a finite frequency band around the central frequency of the measurement, $\nu_{0} \pm \delta \nu$, which is included in equation $(3) . W(\nu)$ is the weight given to a particular frequency within the considered frequency range. We apply a cosine-taper within the frequency band of measurement:

$$
W(\nu)=0.5\left[1+\cos \left(\frac{\pi\left(\nu-\nu_{0}\right)}{\delta \nu}\right)\right] .
$$

The choice of $\delta \nu$ and $W(\nu)$ is made both to mimic the frequency band of measurement and to provide a smooth truncation of $K_{q}$ transverse to the great-circle linking source and receiver (i.e., as a function of $\theta$ ). Reasonable variations of these quantities do not change the results of tomography appreciably. All kernels here are computed relative to the 1-D spherically averaged model PREM (Dziewonski and Anderson, 1981).

The shape of the minor-arc kernel given by equation (3) is shown in Figure 1a, truncated after the seventh sensitivity zone (which we define below). Without the frequency integral, the kernel is somewhat more complicated, as Figure 1b illustrates. The spatial complexity of the kernel has motivated several different simplifications. Some researchers have truncated the kernel at the central lobe of the sensitivity kernel, as seen in Figure 1c. Ritzwoller et al. (2002) approximated the kernel further as a box-car function within the central lobe, as seen in Figure 1d. The motivation for the truncation at the central lobe relates to the oscillatory nature of the sensitivity kernel. Upon area integration, the oscillations in the kernel will tend to destructively interfere.

Figure 2 illustrates the oscillatory nature of the kernels transverse to the great-circle linking the source and receiver and clarifies what is meant by the $n$-th sensitivity zone, Fn. The $n$-th sensitivity zone is the region of the sensitivity kernel between the zero-crossings beginning at the great-circle linking source and receiver. We label the first through seventh sensitivity zones as $F 1$ through $F 7$ in Figure 2, such that F1 is the central lobe of the 
kernel. The frequency integral in equation (3) acts to reduce the amplitude of the sensitivity kernel for the second and higher zones. The amplitude of the sensitivity kernel beyond the seventh zone becomes negligible when the frequency integral is applied. If the kernel retains contributions through the $n$-th sensitivity zone, we refer to the forward operator as the Fntheory. For example, in the F1-theory travel times are computed using only the central lobe of the sensitivity kernel as shown in Figure 1c and the F7-theory corresponds to Figure 1a. We refer to the box-car kernel confined to the central lobe, shown in Figure 1c, as the $\overline{\mathrm{F} 1}$-theory. This nomenclature also holds for major-arc measurements. We discuss later how the choice of the forward theory affects resolution and the results of tomography.

If $\Delta>\pi$ (a major-arc path), $K_{(n, q)}=K_{(2, q)}(\Delta, \theta, \phi, \nu)$. The sensitivity kernel decomposes into three component kernels corresponding to discrete segments of the path: (1) between the source and the antipode of the receiver, (2) between the antipode of receiver and the antipode of the source, and (3) between the antipode of the source and the receiver (Spetzler et al., 2002). Examples of the extent of the first and seventh sensitivity zones for a set of periods are shown in Figure $3 \mathrm{a}$ and $3 \mathrm{~b}$. The kernel for each segment is weighted proportionally to the length of the segment as follows:

$$
\begin{aligned}
K_{(2, q)}(\theta, \phi, \nu)= & \frac{1}{\Delta}\left[(\Delta-\pi) K_{(1, q)}((\Delta-\pi), \theta, \phi, \nu)\right. \\
& +(2 \pi-\Delta) K_{(1, q)}((2 \pi-\Delta), \theta, \phi-\Delta+\pi, \nu)(\Delta-\pi) \\
& \left.+K_{(1, q)}((\Delta-\pi), \theta, \phi-\pi, \nu)\right]
\end{aligned}
$$

An example of a major-arc sensitivity kernel is presented in Figure 3c, plotted similarly to the minor-arc kernels in Figure 1.

Equation (3) for the minor-arc kernel, $K_{1 q}$, is not valid near the source $(\phi \sim 0)$ or receiver $(\Delta-\phi \sim 0)$, where $H \sim 0$. There are corresponding singularities in the major-arc kernel at four points; near the source and receiver and their antipodes. To avoid the singularities, we approximate the sensitivity kernels within a circle centered on each singularity with radius $\lambda\left(\nu_{0}\right) / 4$, where $\lambda=v_{q}\left(\nu_{0}\right) / \nu_{0}$ is the wavelength. Within this region, the sensitivity kernel is simply replaced by its profile in $\theta$ at a distance of $\lambda\left(\nu_{0}\right) / 4$ from the singularity. Finally, the 
kernel is normalized by the condition

$$
\int_{S} K_{q}(\mathbf{r}, T) d S=\Delta R_{0} .
$$

The kernels shown in Figures 1 - 3 have been constructed in this way.

The major-arc sensitivity kernels change systematically with both period and epicentral distance. The widening of the kernel with period is seen in Figure 3. The effect of distance is illustrated in Figure 4. As Figure 5 shows, because of the pinching of the sensitivity kernel near the antipodes of the source and the receiver, the maximum width of the sensitivity kernel does not increase continuously with distance for major-arc measurements. The sensitivity kernel does widen monotonically for minor-arc measurements, achieving a maximum for receivers near the antipode of the source (i.e., $\Delta \sim 180^{\circ}$ ). At epicentral distances between $210^{\circ}$ and $330^{\circ}$, however, the maximum width of the major-arc sensitivity kernel is identical to the minor-arc kernel from $90^{\circ}$ to $150^{\circ}$. There are a number of good reasons to prefer minor-arc travel time measurements to major-arc measurements (e.g., higher signal-to-noise, reduced effect of anelastic attenuation, smaller scattering area, narrower sensitivity zones for epicentral distances less than $90^{\circ}$ ), but it is worth remembering that the width of the sensitivity zone for major-arc measurements relative to minor-arc measurements at distances greater than $90^{\circ}$ is not one of them.

The extension of the sensitivity kernels to major-arc measurements allows us to combine minor- and major-arc data for a joint tomographic inversion of phase speed measurements.

\section{Tomographic Method, Path Density, Resolution}

\subsection{Inversion method}

The joint inversion of minor-arc and major-arc measurements to estimate a two-dimensional map of surface wave speeds follows the tomographic method of Barmin et al. (2001) which is based on ray-theory with ad-hoc smoothing and model-norm constraints to regularize the inversion on a discrete grid at regional or global scales. Ritzwoller et al. (2002) discussed 
the extension of the method to incorporate extended sensitivity kernels through the first sensitivity zone and the method generalizes naturally for sensitivity kernels past the first zone. If $G$ is the forward operator that computes travel time from a map using equation (1), the discretized form of the forward problem is

$$
\delta \mathbf{t}=\mathbf{d}=\mathbf{G m}
$$

The penalty function is a linear combination of weighted data misfit $\left(\chi^{2}\right)$, model roughness, and the amplitude of the perturbation relative to a reference map which when discretized is as follows:

$$
(\mathbf{G m}-\mathbf{d})^{T} \mathbf{C}^{-1}(\mathbf{G m}-\mathbf{d})+\mathbf{m}^{T} \mathbf{Q m},
$$

where $\mathbf{d}$ is the data vector whose components are the observed travel time residuals relative to the reference map and $\mathbf{C}$ is the data covariance matrix or matrix of data weights. Barmin et al. (2001) discuss the form of $\mathbf{m}$ for both isotropic and azimuthally anisotropic inversions. The matrix $\mathbf{Q}$ represents the effect of a Gaussian spatial smoothing operator with standard deviation $\sigma$ (in $\mathrm{km}$ ) as well as an operator that penalizes the norm of the model in regions of poor path coverage. The choice of the trade-off (or regularization) parameters in $\mathbf{Q}$ and the smoothing width $\sigma$ is ad hoc. We typically apply spatial smoothing widths from 150 to $300 \mathrm{~km}$. Even though extended spatial sensitivity kernels naturally regularize the inversion, additional regularization is still needed.

Here, the inverse problem is discretized onto a global $2^{\circ} \times 2^{\circ}$ grid (i.e., $222 \mathrm{~km} \times 222 \mathrm{~km}$ ) In practice, the sensitivity kernel is constructed along the equator, as described above, and is translated and rotated into each source-receiver configuration. For the forward problem, the kernel is constructed on a $1^{\circ} \times 1^{\circ}$ grid.

As discussed in the following sections, details of the results for path density, resolution, and the tomographic maps will depend on the nature and truncation level of the sensitivity kernels (e.g., F1, F7, etc.), as different kernels will produce different travel times. The magnitude of the difference in travel times as a function of epicentral distance can be seen in Figure 6, which is based on the station and event pairs from the cleaned data set discussed in section 4 . The difference in travel times computed with the central lobe forward theories F1 
(Fig. 1c) and $\overline{F 1}$ (Fig. 1d) is negligible. Interestingly, travel times computed with forward theory F7 (Fig. 1a) are more similar to ray theoretic travel times than they are to travel times computed with theory F1. In addition, the agreement between travel times computed with theory F1 and ray theory, on average, is not as good as comparison between theory F7 and ray theory. The addition of sensitivity zones past the first, therefore, moves the computed travel times back towards those computed with ray theory. This is due to destructive interference between the side-lobes and the principal lobe of the sensitivity kernel with forward theory F7. This will be discussed further as the paper progresses.

\subsection{Pseudo-path-density and resolution}

Aspects of the improvement expected in the tomographic maps by introducing major-arc measurements can be summarized by path density and resolution. For "Gaussian tomography" (i.e., ray theory with ad-hoc smoothing), Barmin et al. (2001) defined path density $\rho(\mathbf{r})$ as the number of paths intersecting a square cell centered at point $\mathbf{r}$ with a fixed area of $2^{\circ} \times 2^{\circ}\left(\sim 50,000 \mathrm{~km}^{2}\right)$. For diffraction tomography based on spatially extended sensitivity kernels, this definition is not appropriate because each path is not a linear object. For this reason, we introduce the notion of pseudo-path-density, $\rho_{D}(\mathbf{r}, T)$, by means of the formula:

$$
\rho_{D}(\mathbf{r}, T)=\sum_{n} \tilde{K}_{q}^{n}
$$

where $\tilde{K}_{q}^{n}$ is the smoothed envelope of the sensitivity kernel from equation (1) evaluated at position $\mathbf{r}$ for measurement $n$, renormalized by equation (6). Summation is made over all $n$ measurements for which $\mathbf{r}$ is inside the sensitivity kernel. With this definition, pseudo-pathdensity is similar to ray-theoretic path density in regions of many crossing paths, but the two measures of path density differ is regions of relatively poor path coverage.

The estimator based on equation (7) describing an isotropic map of velocity perturbations is

$$
\hat{\mathbf{m}}=\mathbf{G}^{\dagger} \mathbf{C}^{-1} \delta \mathbf{t}=\left(\mathbf{G}^{\dagger} \mathbf{C}^{-1} \mathbf{G}\right) \mathbf{m}=\mathcal{R} \mathbf{m}
$$


where $\mathbf{G}^{\dagger}$ is the inverse operator

$$
\mathbf{G}^{\dagger}=\left(\mathbf{G}^{T} \mathbf{C}^{-1} \mathbf{G}+\mathbf{Q}\right)^{-1} \mathbf{G}^{T}
$$

and the resolution matrix $\mathcal{R}$ is

$$
\mathcal{R}=\left(\mathbf{G}^{T} \mathbf{C}^{-1} \mathbf{G}+\mathbf{Q}\right)^{-1} \mathbf{G}^{T} \mathbf{C}^{-1} \mathbf{G} .
$$

In this application, each row of $\mathcal{R}$ is a resolution map defining the resolution at one spatial node. The resolution matrix is consequently very large and the information it contains is somewhat difficult to utilize. We summarize the information in each resolution map by estimating a scalar quantity, which we call the spatial resolution at each point of the grid. The spatial resolution is determined here in a slightly different manner than in Barmin et al. (2001). To estimate resolution, we fit a cone near the target node to each resolution map. This cone approximates the response of the tomographic procedure to a $\delta$-like perturbation at the target node. The radius of the base of the cone was taken by Barmin et al. (2001) as the value of the spatial resolution. In many cases, however, the shape of the response more closely resembles a 2-D spatial Gaussian function, and the cone-based estimate is biased to large values. To reduce this bias, we introduce a new estimate of the spatial resolution summarized by the $\gamma$-parameter, the standard deviation of the 2-D symmetric spatial Gaussian function that best-fits the resolution map in the neighborhood of the target node:

$$
A \exp \left(-\frac{|\mathbf{r}|^{2}}{2 \gamma^{2}}\right)
$$

Here, $A$ is the amplitude of the fit-Gaussian at the target node. As a practical matter, to construct the optimal Gaussian function, we take the absolute value of the resolution map and discard as random noise all points of the map with amplitude less than about $A / 10$. Fitting is done within one resolution length defined by the fit-cone method.

\section{Data}

\subsection{Input data and data handling}

An expanded set of surface wave phase speed measurements, originally described by Trampert \& Woodhouse (1995), was used in the tomographic inversion. We limited ourselves to 
two periods, $50 \mathrm{sec}$ and $100 \mathrm{sec}$, and analyzed only Rayleigh wave data at these periods. In what follows, we will refer to the minor-arc Rayleigh wave observations as R1 and the major-arc observations as $R 2$. The number of paths for the raw data set (R1, R2) is given in Table 1 (column 3). We identify outliers with a two-stage process. In the first stage, we computed synthetic travel times using equation (1) with forward theory F7 (Fig. 1a) using the 3-D model of Shapiro \& Ritzwoller (2002) for all paths contained in the raw data. Figure 7 shows the rms relative travel time residuals [(observed - predicted)/observed] for the raw data as a function of distance. The mean values and $\pm 2.5 \times \mathrm{rms}$ in the window sliding along epicentral distance are presented as well. The gaps in the data at epicentral distances from $160^{\circ}-200^{\circ}$ and $340^{\circ}-360^{\circ}$ reflect interference between minor-arc and major-arc wave trains near the epicenter and its antipode. The corresponding values of rms for phase speed residuals averaged over epicentral distance are given in the Table 1 (column 4). Only measurements with a relative residual between $\pm 2.5 \times \mathrm{rms}$ are selected for further analysis. The numbers of selected paths are presented in Table 1 (column 5).

In the second stage of data selection, we apply a consistency test to the measurements that pass the first stage of selection. This test has been described by Ritzwoller \& Levshin (1998), and is referred to as a cluster or summary-ray analysis. The procedure compares measured travel times along paths with end-points that lie within the same $110 \mathrm{~km} \times 110$ $\mathrm{km}$ cell. We delete duplicates and reject inconsistent measurements. After this test, the number of selected paths is reduced substantially as can be seen in Table 1 (column 6). This procedure also allows us to estimate the inherent errors in the measurements. The average rms value for the whole set of close paths with consistent travel times is given in column 7 of Table 1. The relative rms-misfit for the R2 phase velocities are slightly lower than for R1 due to the greater lengths of the wave paths, although the absolute travel time misfit grows with epicentral distance, as Figure 8 shows, except at distances between about $125^{\circ}$ and $225^{\circ}$ where there is significant growth of rms. This may indicate difficulty in measuring phase speeds accurately due to interference between R1 and R2 waves or interference with Love waves. The general increase of the travel time residuals with distance may be partly due to the systematic decrease of the signal-to-noise ratio. One way to reduce the effect 
of noise is to introduce data weighting inversely proportional to some power of distance in the inversion procedure. We prefer here not to apply this weighting as there is the evident danger of losing the R2 signal.

\subsection{Pseudo-path-density and resolution}

The Pacific Ocean and Antarctic regions are relatively poorly covered by minor-arc observations due to a coarse network of observing stations in these regions. Adding major-arc observations is particularly important for these regions. The left side of Figure 9 shows several views of the pseudo-path-density for the $50 \mathrm{sec}$ Rayleigh wave with only minor-arc data. The right side of the same figure demonstrates the path density for major-arc data. The two distributions are complementary, particularly across the Pacific. Addition of major-arc measurements is expected to have the biggest effect in the South Pacific, Antarctica, Africa, and the Indian Ocean. Path densities for 100 sec surface waves have a similar pattern.

Figure 10 presents several views of the spatial resolution obtained with minor-arc data alone and contrasts the result with the resolution obtained with a combination of minor-arc and major-arc data for $50 \mathrm{sec}$ surface waves. The addition of the major-arc measurements significantly improves the resolution across the Pacific and Antarctica. In regions such as Eurasia and North America that are well covered by minor-arc measurements, little change in resolution results from the addtion of major-arc measurements. A similar pattern is obtained for the $100 \mathrm{sec}$ surface waves.

\section{Results of Tomographic Inversion}

The results of the tomographic inversion of the combined minor-arc and major-arc data $[\mathrm{R} 1+\mathrm{R} 2]$ for Rayleigh waves at periods of 50 and $100 \mathrm{sec}$ are shown in Figures 11 and 12. For comparison, the results based on the minor-arc data alone are also presented. The absolute value of the difference between these maps is shown in Figure 12. As expected, the changes are small in the northern hemisphere where path coverage with minor-arc data is relatively good. Both the amplitudes and the length-scales of the differences are small. There 
is no large scale systematic pattern of difference. Larger amplitude and more systematic differences are observed across much of the southern hemisphere. To quantify this northsouth discrepancy further, we compare the maps in the two polar caps: $45^{\circ} N-90^{\circ} N$ and $45^{\circ} S-90^{\circ} S$. The northern polar cap is relatively well covered by $\mathrm{R} 1$ paths, but much of the southern cap is poorly covered. Table 2 shows the correlation between the maps constructed with major-arc and minor-arc data $(R 1+R 2)$ with those constructed with minor-arc data alone $(R 1)$ at periods of 50 and $100 \mathrm{sec}$ in these two regions. For the northern polar cap, the correlation between the maps produced with the two data sets is much better than in the southern cap and the rms of the absolute difference between the two maps is about $2 / 3$ of the difference in the southern polar cap.

We have shown, therefore, that the introduction of major-arc measurements improves data coverage and resolution across much of the southern hemisphere and also substantially affects the tomographic maps themselves. There is little effect in regions that are well covered by minor-arc data. But are the maps that result from the simultaneous inversion of majorarc and minor-arc data improved relative to maps derived from the minor-arc data alone? By improvement, we mean more accurate and with more detailed information on the phase speed distribution across the globe. Specifically, because the major-arc measurements are noisier than the minor-arc measurements, does their inclusion merely increase the noise in the estimated maps?

One way to address this question is to examine the difference between the fit to the minor-arc data both from maps obtained from the minor-arc data alone and from maps based on both major-arc and minor-arc measurements. If major-arc data can be introduced without appreciably degrading the fit to the minor-arc measurements, then there is good reason to include the major-arc data. If the fit to the minor-arc measurements is degraded strongly, then one may wish not to take on the risk of introducing the more noisy major-arc measurements.

Table 3 contains information about misfit between observed and predicted travel times and phase speeds for different combinations of Rayleigh wave maps and data sets across the whole Earth. The 50 sec Rayleigh wave phase speed map produced from the combination 
of minor-arc and major-arc data $(R 1+R 2)$ only slightly decreases the fit to observations of the minor-arc data, from 9.5 to $10.3 \mathrm{sec}$. The fit to the major-arc measurements with the $R 1+R 2$ map, however, is considerably better than the fit to these measurements with the map constructed with minor-arc data alone $(R 1): 20.9$ sec versus 27.6 sec. A similar result holds at $100 \mathrm{sec}$ period. This indicates that the addition of major-arc data does not significantly degrade the map in regions where minor-arc data exist. Elimination of these data, however, ensures that the major-arc measurements will not be well fit by data based on minor-arc measurements alone.

The tomographic results presented here (Fig. 11 - 13) are for the F7 sensitivity kernels which extend out through the seventh sensitivity zone (e.g., Fig. 1a). The results are similar if we had used the F1 sensitivity zone (e.g., Fig. 1c); i.e., if we had truncated the kernel at the central lobe of the sensitivity kernel. Figure 14 compares the $50 \mathrm{sec}$ Rayleigh wave phase speed maps estimated with the F1 and F7 sensitivity zones. The rms of the differences globally is about $18 \mathrm{~m} / \mathrm{sec}$, or less than $0.5 \%$. The difference between the maps estimated with the two variants of the sensitivity kernels truncated at the central lobe, theories F1 and $\overline{F 1}$, is even smaller with a global rms differences of about $4 \mathrm{~m} / \mathrm{sec}$ or less than $0.1 \%$. Differences between maps derived from theories $\mathrm{F} 1$ and $\overline{F 1}$ are smaller than differences that arise from arbitrary changes in the damping parameters that drive the inversion and are, therefore, negligible. Although the effective difference between theories F1 and F7 is also small, for reasons we discuss in section 6 we prefer and advise the use of theory F7 over theories $\mathrm{F} 1$ or $\overline{F 1}$ unless epicentral distances are well less than $90^{\circ}$.

\section{Discussion and Conclusions}

We have shown that the introduction of major-arc surface wave dispersion measurements improves path density and resolution in regions poorly covered by minor-arc measurements alone, as occurs in much of the southern hemisphere. In addition, we showed that majorarc measurements can be added to the inversion for dispersion maps without appreciably degrading the fit to the minor-arc measurements but significantly improving the fit to the 
major-arc measurements. For these reasons, we conclude that the addition of major-arc measurements is worthwhile as an interim solution until the broad-band network of ocean bottom or Antarctic stations is improved in the future.

The addition of major-arc measurements comes with a cost, however. The measurements are noisier than minor-arc measurements and major-arc sensitivity kernels are broad, complicated spatial functions. Analysis of misfit implies that the reduction of signal-to-noise in the major-arc measurements does not mitigate against their inclusion in the inversion. Although ray theoretic travel times may be sufficiently accurate for epicentral distances less than $60^{\circ}$ - $90^{\circ}$, the ray theoretic approximation degrades rapidly for longer minor-arc distances and for major-arc measurements.

Although we advocate using sensitivity kernels beyond the central lobe, computational expedience may dictate a more approximate method to compute travel times and sensitivity. The use of all or some fraction of the central lobe is popular (e.g., Yoshizawa and Kennett, 2002, Ritzwoller et al., 2002). The central lobe of the sensitivity kernel is commonly identified as the first Fresnel zone, which is an ellipse on a sphere given by the the equation

$$
\left|\Delta-\left(\Delta_{1}+\Delta_{2}\right)\right|=\lambda / N
$$

as shown in Figure 15, where $\lambda$ is the wavelength of the wave of interest determined from PREM here. By comparing the maximum width of the central lobe of the sensitivity kernel to the width of the first Fresnel zone, Spetzler et al. (2002) showed that $N=8 / 3$. Ritzwoller et al. (2002) used this value of $N$ to perform global tomography in which the sensitivity kernel was confined to the central lobe and shaped like a box-car (i.e., theory $\overline{F 1}$ shown in Fig. 1d). Yoshizawa and Kennett (2002) argue that the "zone of influence" about surface wave paths over which the surface waves are coherent in phase is considerably narrower than the first Fresnel zone, being only about one-third of the width of the first Fresnel zone and, consistent with this, a better choice for $N$ in equation (14) is $N=18$.

Aspects of the results presented here corroborate the arguments of Yoshizawa and Kennett (2002). For example, Figure 6 shows that except near the source antipode, ray theoretic travel times agree better with F7-theory (i.e., in which the sensitivity kernel extends through 
the seventh sensitivity zone) than the agreement between F1-theory with F7-theory. This is because of destructive interference among the side-lobes and with the central lobe of the sensitivity kernel. Similarly, the resolution of tomography produced with F7-theory is better than that with F1-theory as shown in Figure 16. This is on first sight counter-intuitive, that a spatially broader sensitivity kernel would improve resolution. But, again, it is because of destructive interference between the side-lobes and the central lobe. The result is to produce a sensitivity kernel that, in effect, is narrower than the first Fresnel zone. If one wishes to utilize a sensitivity kernel that includes only the central lobe, our results suggest to narrow the central lobe as Yoshizawa and Kennett argue.

\section{Acknowledgements}

We are grateful to Nikolai Shapiro, Jesper Spetzler, Ken Creager and an anonymous reviewer for constructive comments. All maps were generated with the Generic Mapping Tools (GMT) data processing and display package (Wessel and Smith, 1991, 1995). This work was supported by two grants from the US National Science Foundation: EAR-0337622 and OPP-0125848. 


\section{References}

Barmin, M.P., A.L. Levshin, and M.H. Ritzwoller, 2001. A fast and reliable method for surface wave tomography, Pure Appl. Geophys., 158: 1351-1375.

Bostock, M.G. and B.L.N. Kennett, 1992. Multiple scattering of surface waves from discrete obstacles, Geophys. J. Int., 108: 52-70.

Dziewonski, A. M. and D. L. Anderson, 1981. Preliminary Reference Earth Model, Phys. Earth. Planet. Int., 25: 297-356.

Friederich, W., E. Wielandt, and S. Strange, 1993. Multiple forward scattering of surface waves; Comparison with an exact solution and the Born single-scattering methods, Geophys. J. Int., 112: 264-275.

Friederich, W., 1999. Propagation of seismic shear and surface waves in a laterally heterogeneous mantle by multiple forward scattering, Geophys. J. Int., 136: 180-204.

Levin, V., N.M. Shapiro, J. Park, and M.H. Ritzwoller, 2002. Seismic evidence for catastrophic slab loss beneath Kamchatka, Nature, 418: 763-767.

Levshin, A.L., M.H. Ritzwoller, M.P. Barmin, A. Villaseñor, 2001. New constraints on the Arctic crust and uppermost mantle: Surface wave group velocities, $P_{n}$, and $S_{n}$, Phys. Earth. Planet. Int., 123: 185-204.

Meier, T., S. Lebedev, G. Nolet, and F.A. Dahlen, 1997. Diffraction tomography using multimode surface waves, J. Geophys. Res., 102(B4): 8255-8267.

Ritzwoller, M.H. and A.L. Levshin, 1998. Eurasian surface wave tomography: Group velocities, J. Geophys. Res., 103: 4839-4878.

Ritzwoller, M.H., N.M. Shapiro, A.L. Levshin, and G.M. Leahy, 2001. Crustal and upper mantle structure beneath Antarctica and surrounding oceans, J. Geophys. Res., 106(B12): 30,645-30,670. 
Ritzwoller, M.H., N.M. Shapiro, M.P. Barmin, and A.L. Levshin, 2002. Global surface wave diffraction tomography, J. Geophys. Res., 107(B12): 2335, doi:10.1029/2002JB001777.

Ritzwoller, M.H., N.M. Shapiro, and G.M. Leahy, 2003a. A resolved mantle anomaly as the cause of the Australian-Antarctic Discordance, J. Geophys. Res., 108(B12): 2559, doi:10.1029/2003JB002522.

Ritzwoller, M.H., N.M. Shapiro, A.L. Levshin, E.A. Bergman, and E.R. Engdahl, 2003b. The ability of a global 3-D model to locate regional events, J. Geophys. Res., 108(B7): 2353, ESE 9-1 - ESE 9-24.

Ritzwoller, M.H., N.M. Shapiro, S. Zhong, 2004. Cooling history of the Pacific lithosphere, Earth Planet. Sci. Letts., submitted.

Shapiro, N.M. and M.H. Ritzwoller, 2002. Monte Carlo inversion for a global shear velocity model of the crust and upper mantle, Geophys. J. Int., 151: 88-105.

Snieder, R. and B. Romanowicz, 1988. A new formalism for the effect of lateral heterogeneity on normal modes and surface waves - I: Isotropic perturbations, perturbations of interfaces and gravitational perturbations, Geophys. J. R. astr. Soc, 92, 207-222.

Snieder, R., 2002. Scattering of surface waves, in Scattering and Inverse Scattering in Pure and Applied Science, eds. R. Pike and P. Sabatier, Academic Press, San Diego: $562-577$.

Spetzler, J., J. Trampert, and R. Snieder, 2001. Are we exceeding the limits of the great circle approximation in global surface wave tomography?, Geophys. Res. Let. 28(12): 2341-2344.

Spetzler, J., J. Trampert, and R. Snieder, 2002. The effect of scattering in surface wave tomography, Geophys. J. Int., 149: 755-767.

Trampert, J., and J. Woodhouse, 1995. Global phase velocity maps of Love and Rayleigh waves between 40 and 150 seconds, Geophys. J. Int., 122: 675-690. 
Trampert, J., and J. Woodhouse, 1996. High resolution global phase velocity distributions. Geophys. Res. Lett., 23: 21-24.

Trampert, J., and J. Woodhouse, 2003. Global anisotropic phase velocity maps for fundamental mode waves between 40 and 150 seconds, Geophys. J. Int., 154: 154-165.

van Heijst, H.J. \& Woodhouse, J.H., 1999. Global high-resolution phase velocity distribution of overtone and fundamental-model surface waves determined by mode branch stripping, Geophys. J. Int., 137: 601-620.

Wang, Z. and F.A. Dahlen, 1995. Validity of surface-wave ray theory on a laterally heterogeneous earth, Geophys. J. Int., 123: 757-773.

Wessel, P., and W.H.F. Smith, 1991. Free software helps map and display data, EOS, 72: 441.

Wessel, P., and W.H.F. Smith, 1995. New version of the Generic Mapping Tools released, EOS, 76: 329 .

Woodhouse, J.H., 1974. Surface waves in a laterally varying layered structure, Geophys. J. R. astr. Soc., 37: 461-490.

Woodhouse, J. H. and T. P. Girnius, 1982. Surface waves and free oscillations in a regionalized Earth model, Geophys. J. R. astr. Soc., 68: 653-673.

Yomogida, K., and K. Aki, 1987. Amplitude and phase data inversion for phase velocity anomalies in the Pacific Ocean basin, Geophys. J. R. astr. Soc., 88, 161-204.

Yoshizawa, K. and B.L.N. Kennett, 2002. Determination of the influence zone for surface wave paths, Geophys. J. Int., 149: 440-453. 
Table 1. Number of measurements before and after each of the two stages of the data selection procedure.

\begin{tabular}{|c|c|c|c|c|c|c|}
\hline \hline $\begin{array}{c}\text { Period } \\
\text { sec }\end{array}$ & $\begin{array}{c}\text { Wave } \\
\text { Type }\end{array}$ & $\begin{array}{c}\text { Number of } \\
\text { Input Paths }\end{array}$ & $\begin{array}{c}\text { Rms, Ph. Vel. } \\
\text { Res., m/s }\end{array}$ & $\begin{array}{c}\text { Number of Selected } \\
\text { Paths (1st Stage) }\end{array}$ & $\begin{array}{c}\text { Number of Selected } \\
\text { Paths (2nd Stage) }\end{array}$ & $\begin{array}{c}\text { Rms, Ph. Vel. } \\
\text { Errors, m/s }\end{array}$ \\
\hline \hline 50 & R1 & 54168 & 22 & 48192 & 27310 & 19 \\
\hline 50 & R2 & 21347 & 27 & 17476 & 12654 & 15 \\
\hline \hline 100 & R1 & 54168 & 26 & 49888 & 26852 & 21 \\
\hline 100 & R2 & 21347 & 30 & 17477 & 13631 & 12 \\
\hline \hline
\end{tabular}

Table 2. Comparison between tomographic maps for the north and south polar caps obtained with minor-arc (R1) and major-arc plus minor-arc (R1 + R2) data sets.

\begin{tabular}{|c|c|c|c|}
\hline \hline Region & $\begin{array}{c}\text { Period } \\
\mathrm{s}\end{array}$ & $\begin{array}{c}\text { Correlation } \\
\text { Coefficient }\end{array}$ & $\begin{array}{c}\text { Rms of difference } \\
\mathrm{m} / \mathrm{s}\end{array}$ \\
\hline \hline $45^{\circ}-90^{\circ} N$ & 50 & 0.969 & 20 \\
\hline $45^{\circ}-90^{\circ} N$ & 100 & 0.966 & 20 \\
\hline \hline $45^{\circ}-90^{\circ} S$ & 50 & 0.938 & 28 \\
\hline $45^{\circ}-90^{\circ} S$ & 100 & 0.893 & 29 \\
\hline \hline
\end{tabular}


Table 3. Misfit between predicted and observed travel times and phase speeds for data from the whole Earth.

\begin{tabular}{|c|c|c|c|c|c|c|}
\hline $\begin{array}{l}\text { Period } \\
\quad \mathrm{s}\end{array}$ & Map & Type of Data & $\begin{array}{l}\text { Number } \\
\text { of Paths }\end{array}$ & $\begin{array}{c}\text { Rms (travel time) } \\
\text { s }\end{array}$ & $\begin{array}{c}\text { Variance }^{\dagger} \\
\text { Reduction, } \%\end{array}$ & $\begin{array}{c}\text { Rms (phase } \\
\text { velocity), } \mathrm{m} / \mathrm{s}\end{array}$ \\
\hline 50 & $\mathrm{R} 1+\mathrm{R} 2$ & $\mathrm{R} 1+\mathrm{R} 2$ & 39964 & 14.5 & 42.4 & 16.3 \\
\hline 50 & $\mathrm{R} 1+\mathrm{R} 2$ & $\mathrm{R} 1$ & 27310 & 10.3 & 13.8 & 18.3 \\
\hline 50 & $\mathrm{R} 1+\mathrm{R} 2$ & $\mathrm{R} 2$ & 12654 & 20.9 & 51.0 & 10.8 \\
\hline 50 & $\mathrm{R} 1$ & $\mathrm{R} 1$ & 27310 & 9.5 & 28.0 & 16.6 \\
\hline 50 & $\mathrm{R} 1$ & $\mathrm{R} 2$ & 12654 & 27.6 & 18.2 & 14.0 \\
\hline 100 & $\mathrm{R} 1+\mathrm{R} 2$ & $\mathrm{R} 1+\mathrm{R} 2$ & 40483 & 12.5 & 32.4 & 17.4 \\
\hline 100 & $\mathrm{R} 1+\mathrm{R} 2$ & $\mathrm{R} 1$ & 26852 & 9.4 & 10.9 & 20.3 \\
\hline 100 & $\mathrm{R} 1+\mathrm{R} 2$ & $\mathrm{R} 2$ & 13631 & 17.0 & 40.9 & 9.3 \\
\hline 100 & $\mathrm{R} 1$ & $\mathrm{R} 1$ & 26852 & 8.8 & 22.8 & 19.0 \\
\hline 100 & $\mathrm{R} 1$ & $\mathrm{R} 2$ & 13631 & 23.3 & -10.6 & 12.9 \\
\hline
\end{tabular}

$\dagger$ Variance reduction is relative to predicted velocities from Shapiro \& Ritzwoller (2002). 

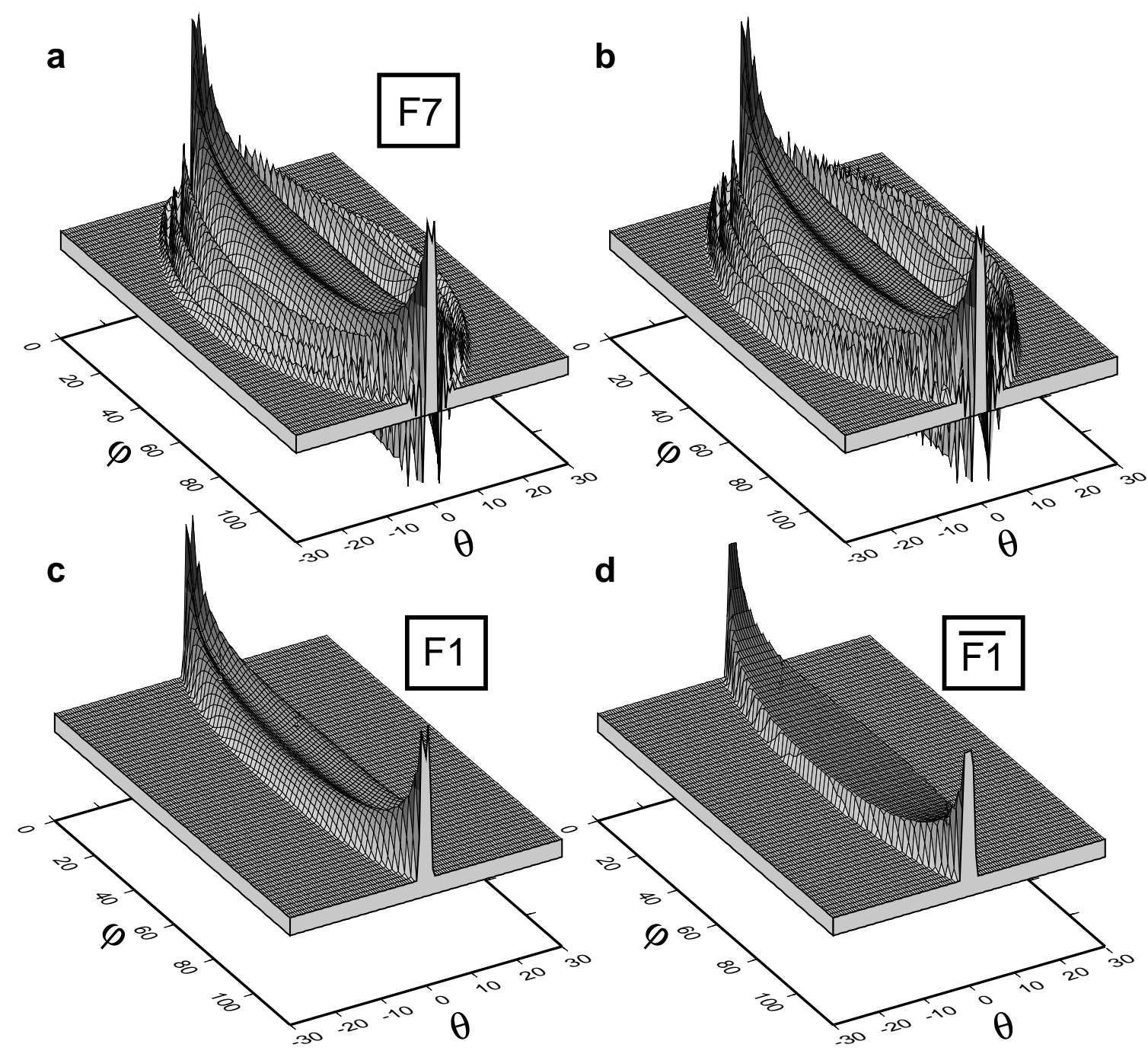

Figure 1: Minor-arc sensitivity kernels for the 50 sec Rayleigh wave phase speed between a source and receiver at coordinates $(\theta, \phi)$ of $(0,0)$ and $(0,120)$; i.e., an epicentral distance $\Delta=120^{\circ}$. (a) The kernel defined by equation (3) is shown, including the frequency integral, truncated after sensitivity zone F7. Referred to as forward theory F7. (b) The same as (a), but the frequency integral has not been performed. (c) The sensitivity kernel truncated at the central lobe of the sensitivity kernel, F1, referred to as forward theory F1. (d) Box-car shaped kernel truncated at the central lobe of the sensitivity kernel (e.g., Ritzwoller et al., $2002)$, referred to as forward theory $\overline{F 1}$. 


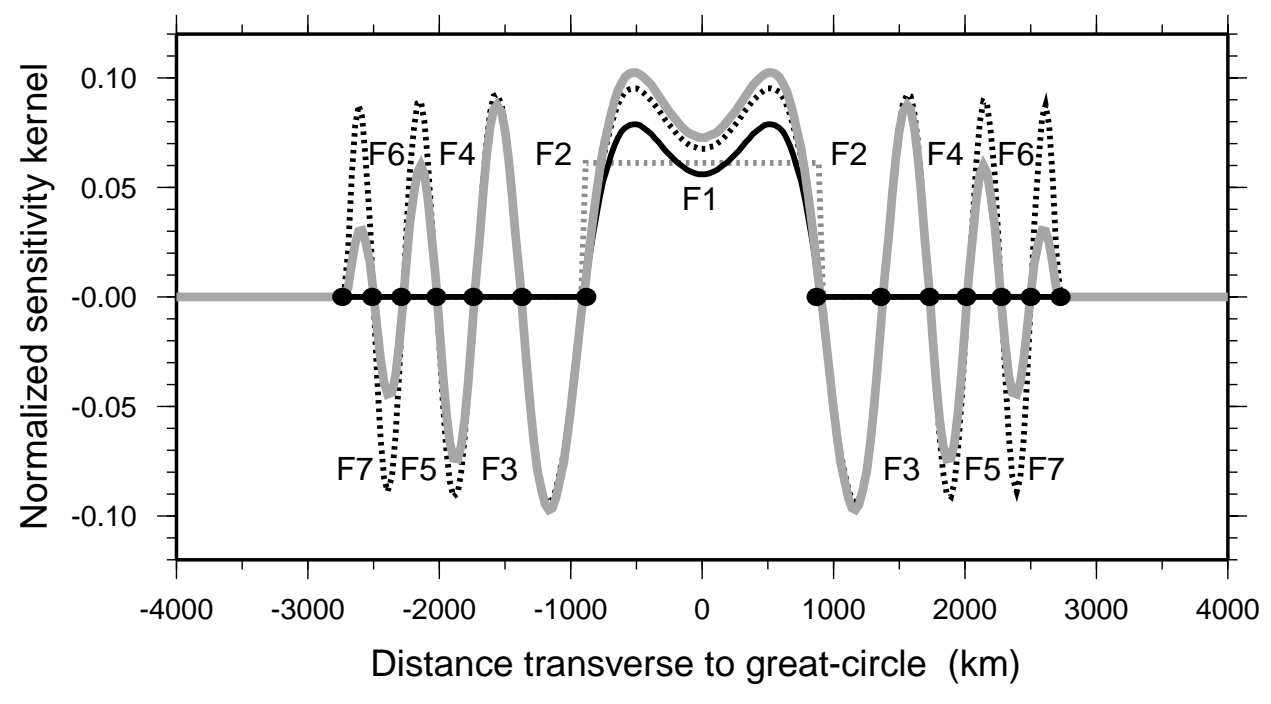

Figure 2: Amplitude of the sensitivity kernels shown in Figure 1 transverse to the great-circle linking the source and receiver. The solid grey line corresponds to Figure 1a, the dashed black line to Figure 1b, the solid black line to Figure 1c, and the dashed grey line to Figure 1d. The zones of sensitivity are defined between the zero crossings of the sensitivity kernel, denoted as F1 for the central lobe of the kernel through F7 for the seventh zone, as shown. 

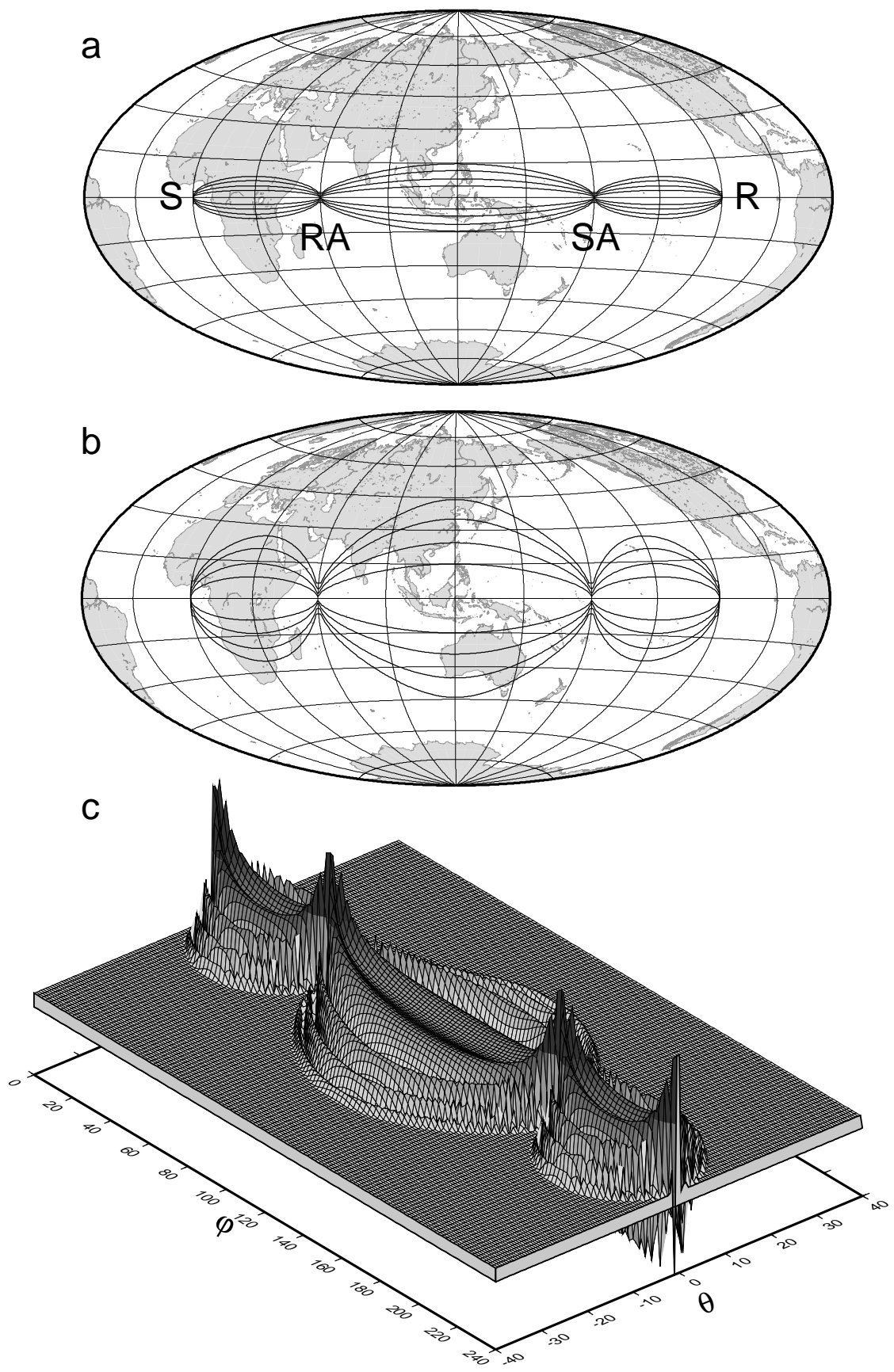

Figure 3: Spatial extent and shape of the major-arc sensitivity kernel for Rayleigh wave phase speeds plotted for several periods at an epicentral distance of $240^{\circ}$. (a) The extent of the central lobe of the sensitivity kernel, F1, is shown for the $20 \mathrm{sec}, 50 \mathrm{sec}, 100 \mathrm{sec}$, and $150 \mathrm{sec}$ Rayleigh waves. The source location $(\mathrm{S})$, the receiver location $(\mathrm{R})$, the source antipode $(\mathrm{SA})$, and the receiver antipode (RA) are indicated. The sensitivity zone widens as period increases. (b) Similar to (a), but this is the extent of the seventh sensitivity zone, F7, plotted for the same periods as in (a). (c) Major-arc sensitivity kernel plotted similarly to the minor-arc kernels shown in Figure 1 for the 50 sec Rayleigh wave phase speed. 

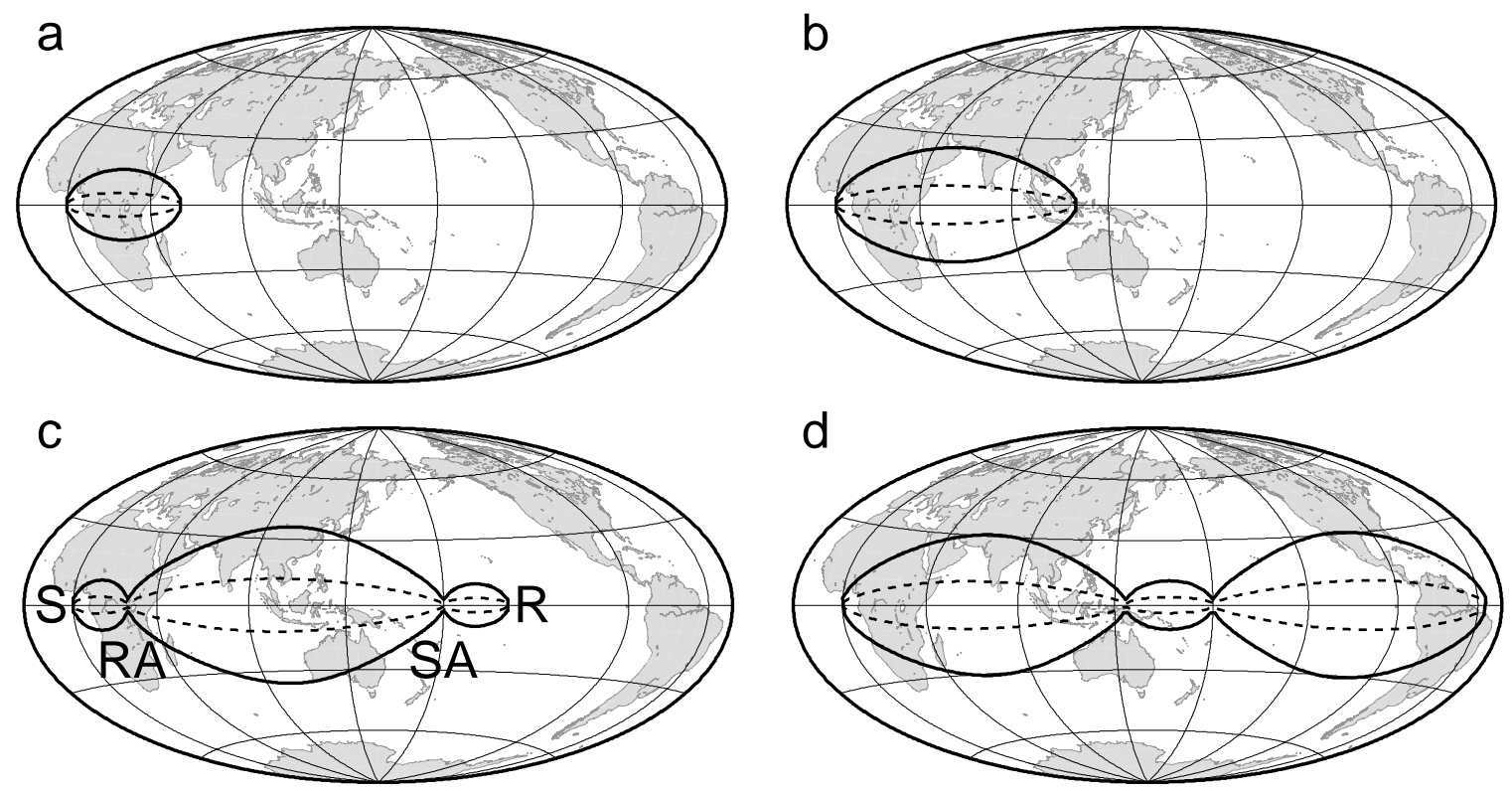

Figure 4: Spatial extent of the sensitivity kernels plotted for the 50 sec Rayleigh wave phase speed at several epicentral distances: (a) $60^{\circ}$, (b) $120^{\circ}$, (c) $210^{\circ}$, and (d) $320^{\circ}$. The dashed lines show the extent of the central lobe of the sensitivity kernel, and the solid lines show the extent of the seventh sensitivity zone. The locations of the source (S), receiver (R), source antipode (SR), and receiver antipode (RA) are shown in (c). 


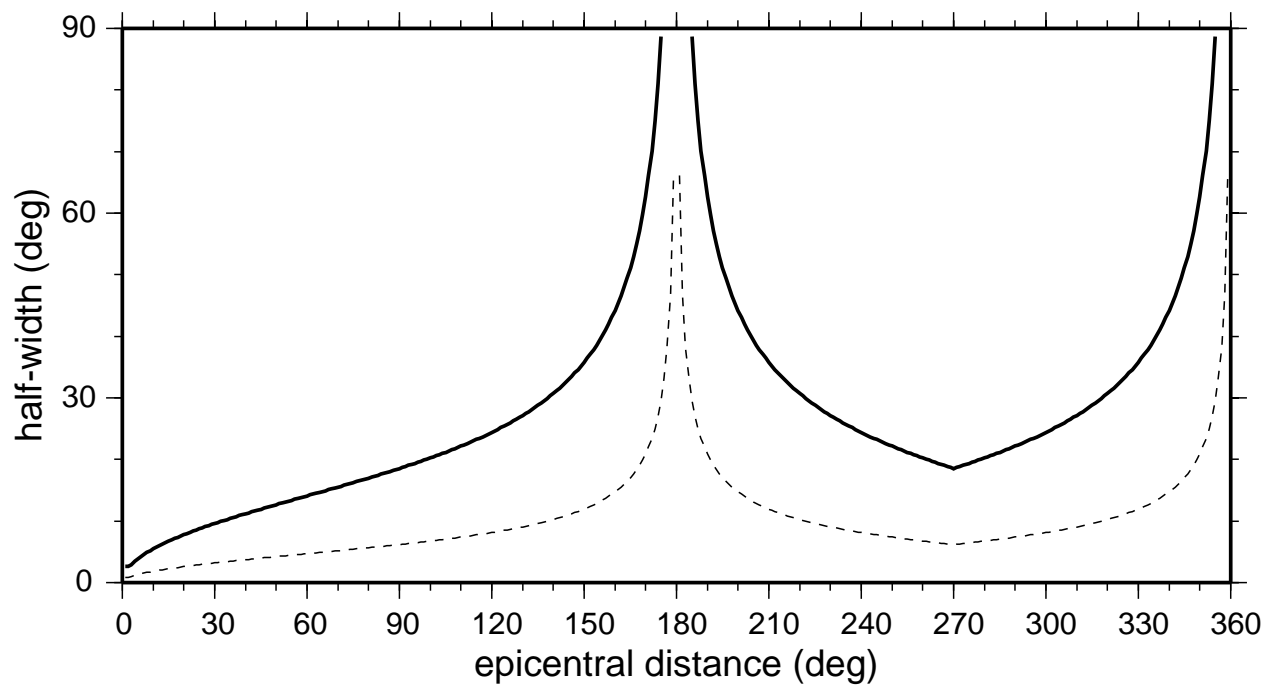

Figure 5: Half the maximum width of the sensitivity kernel for the 50 sec Rayleigh phase speed, plotted as a function of epicentral distance (except near $180^{\circ}$ and $360^{\circ}$ ). The dashed line denotes the edge of the central lobe of the sensitivity kernel, F1, and the solid line the edge of the seventh zone, F7. 


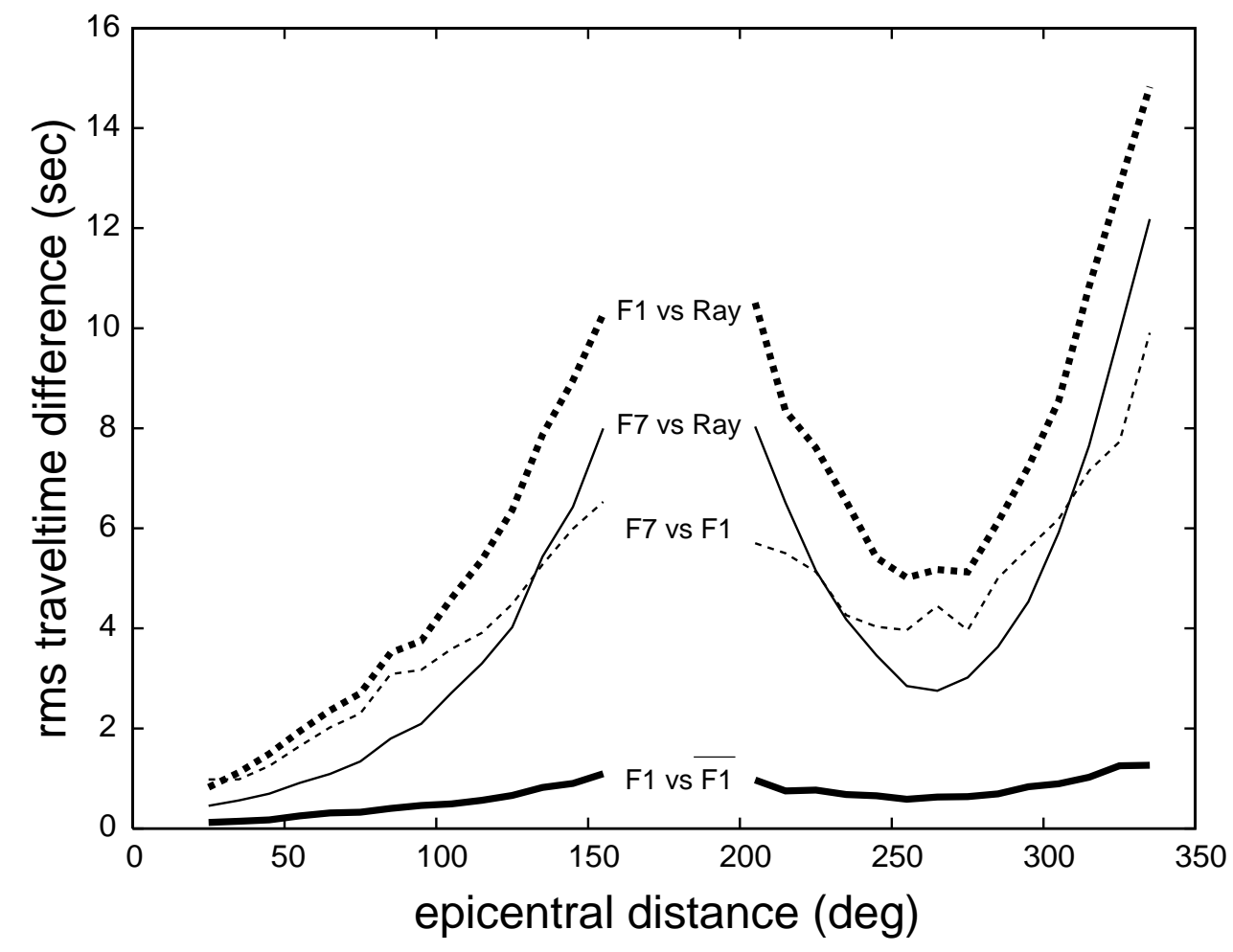

Figure 6: RMS of the difference in synthetic travel times between various forward theories of travel time computation for the 100 sec Rayleigh wave phase speed map computed from the 3-D model of Shapiro and Ritzwoller (2002). The station and event locations used are those from the final, cleaned data set used for tomography. "Ray" denotes ray theoretic travel times and the notation F7, F1, and $\overline{\mathrm{F} 1}$ refers to the sensitivity kernels illustrated in Figure $1 \mathrm{a}, 1 \mathrm{c}$, and $1 \mathrm{~d}$, respectively. 


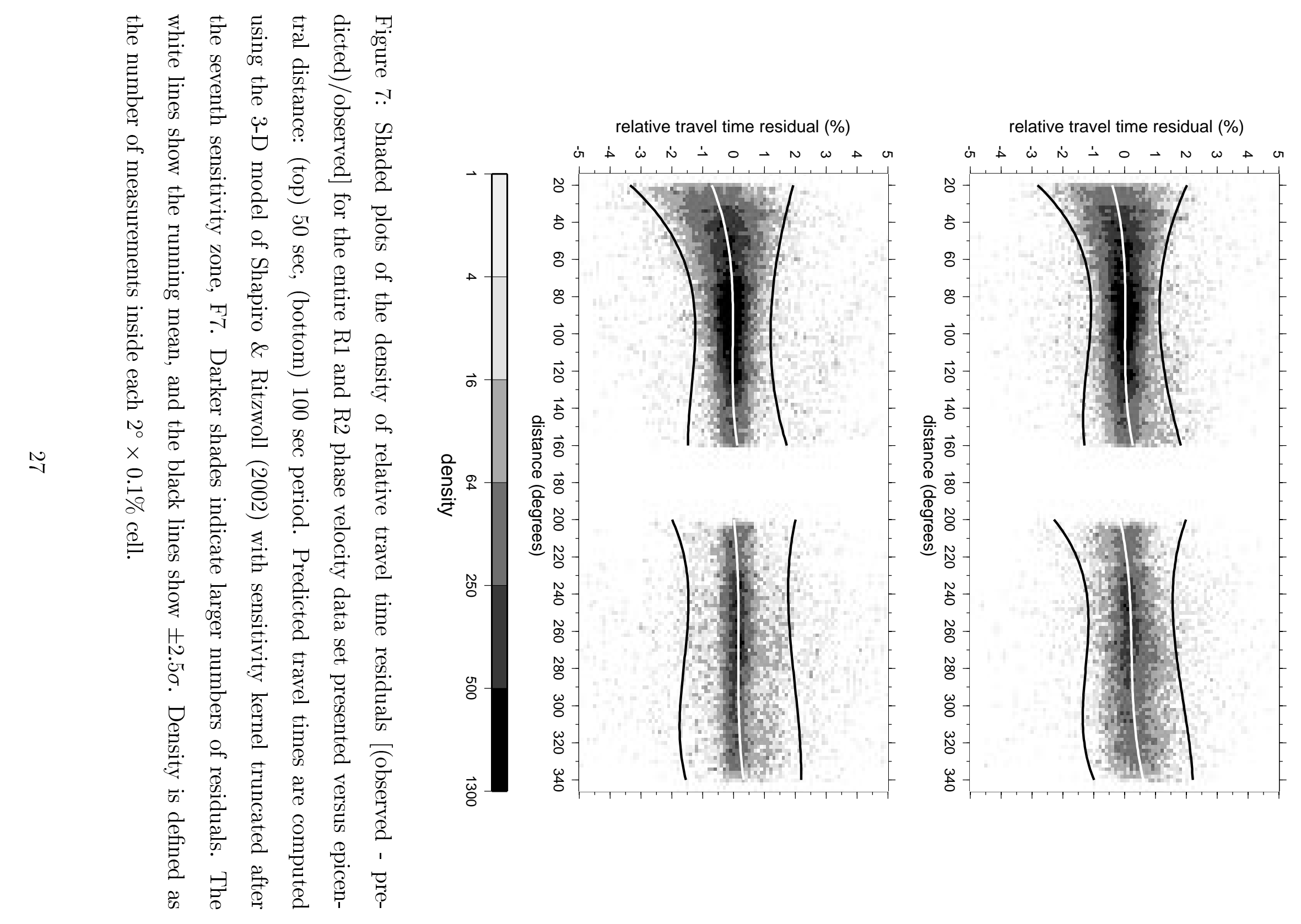




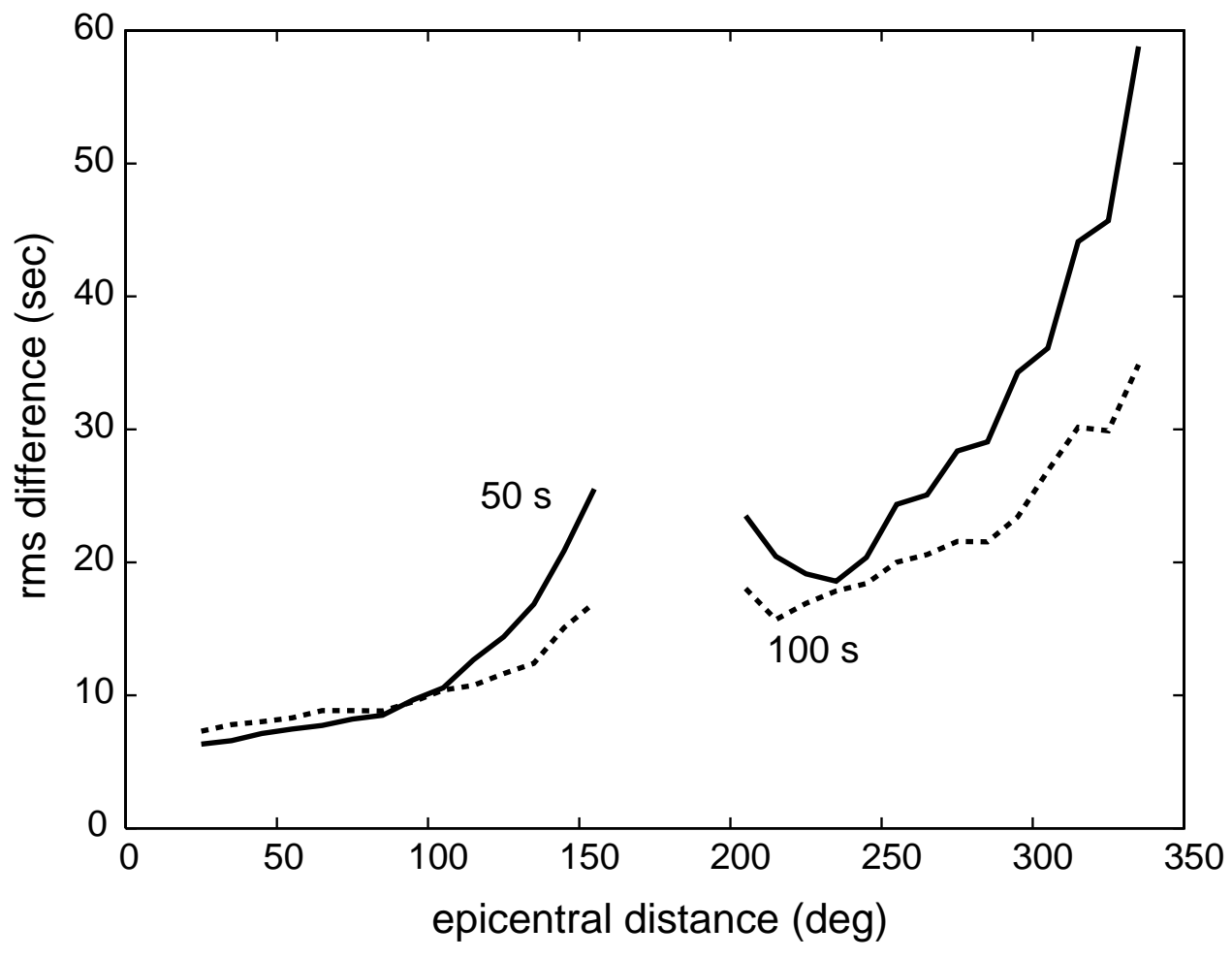

Figure 8: Rms of the travel time residuals with respect to predictions from the 3-D model of Shapiro and Ritzwoller (2002) for the cleaned data set plotted as a function of epicentral distance for $50 \mathrm{sec}$ (solid line) and $100 \mathrm{sec}$ (dashed line) Rayleigh waves. 


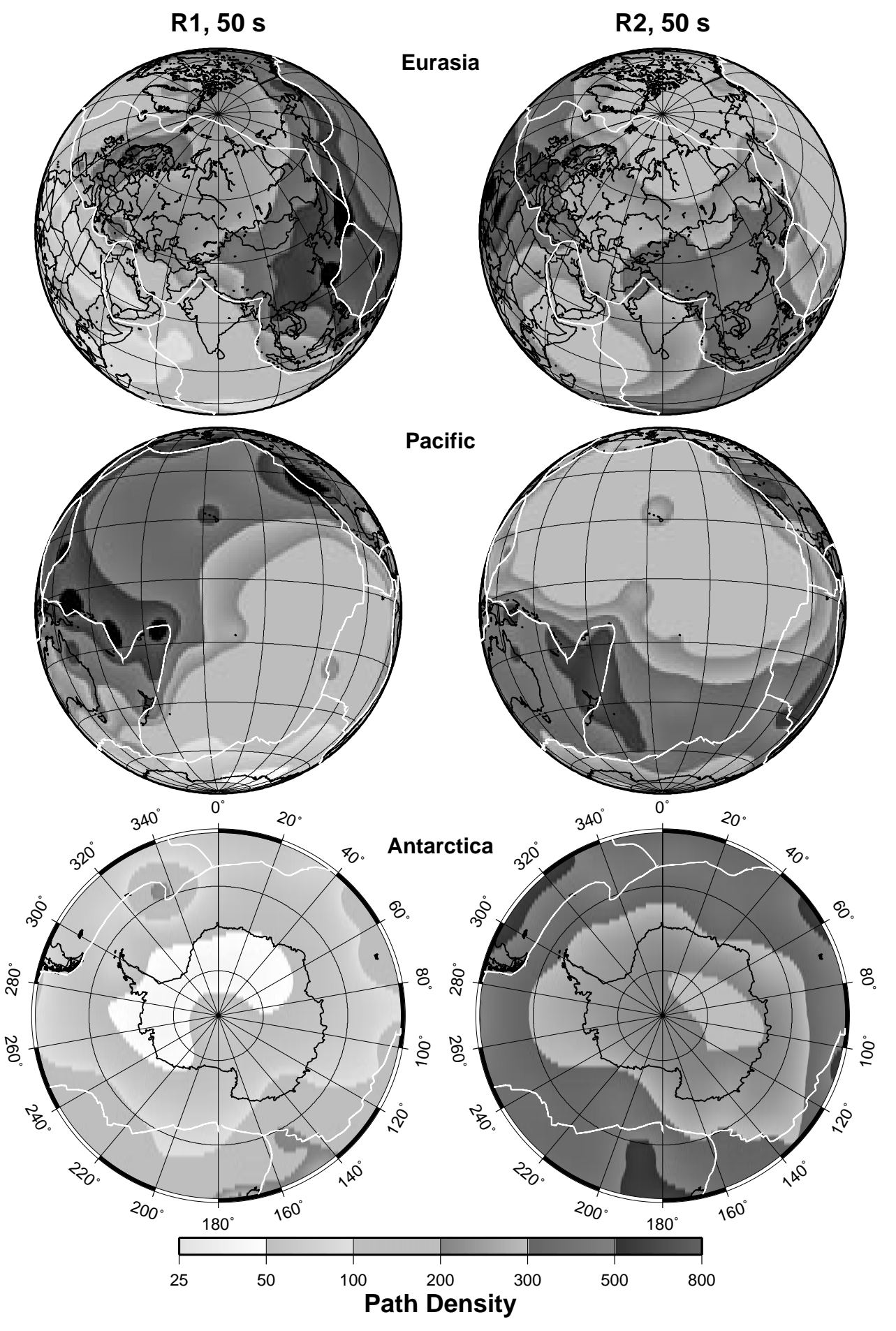

Figure 9: Pseudo-path-density of $50 \mathrm{sec}$ Rayleigh waves: (LEFT) minor-arc data alone, (RIGHT) major-arc data alone. Pseudo-path-density approximates the number of the rays in each $2^{\circ} \times 2^{\circ}$ cell $\left(\sim 50,000 \mathrm{~km}^{2}\right)$. Results are based on the F7 sensitivity kernels (Fig. 1a). 


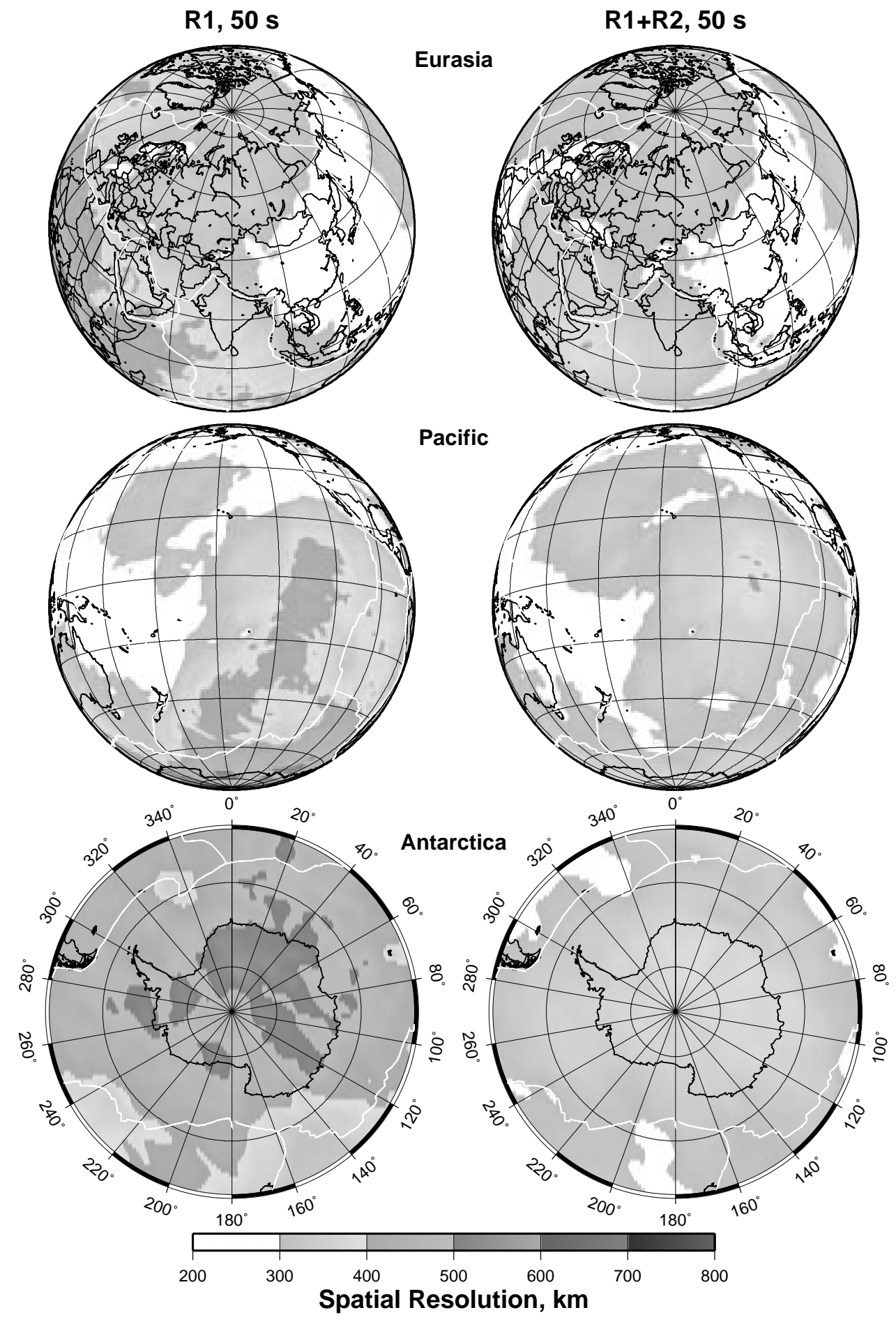

Figure 10: Spatial resolution of 50 sec Rayleigh wave tomography: (LEFT) minor-arc data alone, minor-arc and major-arc data together. Results are based on the F7 sensitivity kernels (Fig. 1a). 


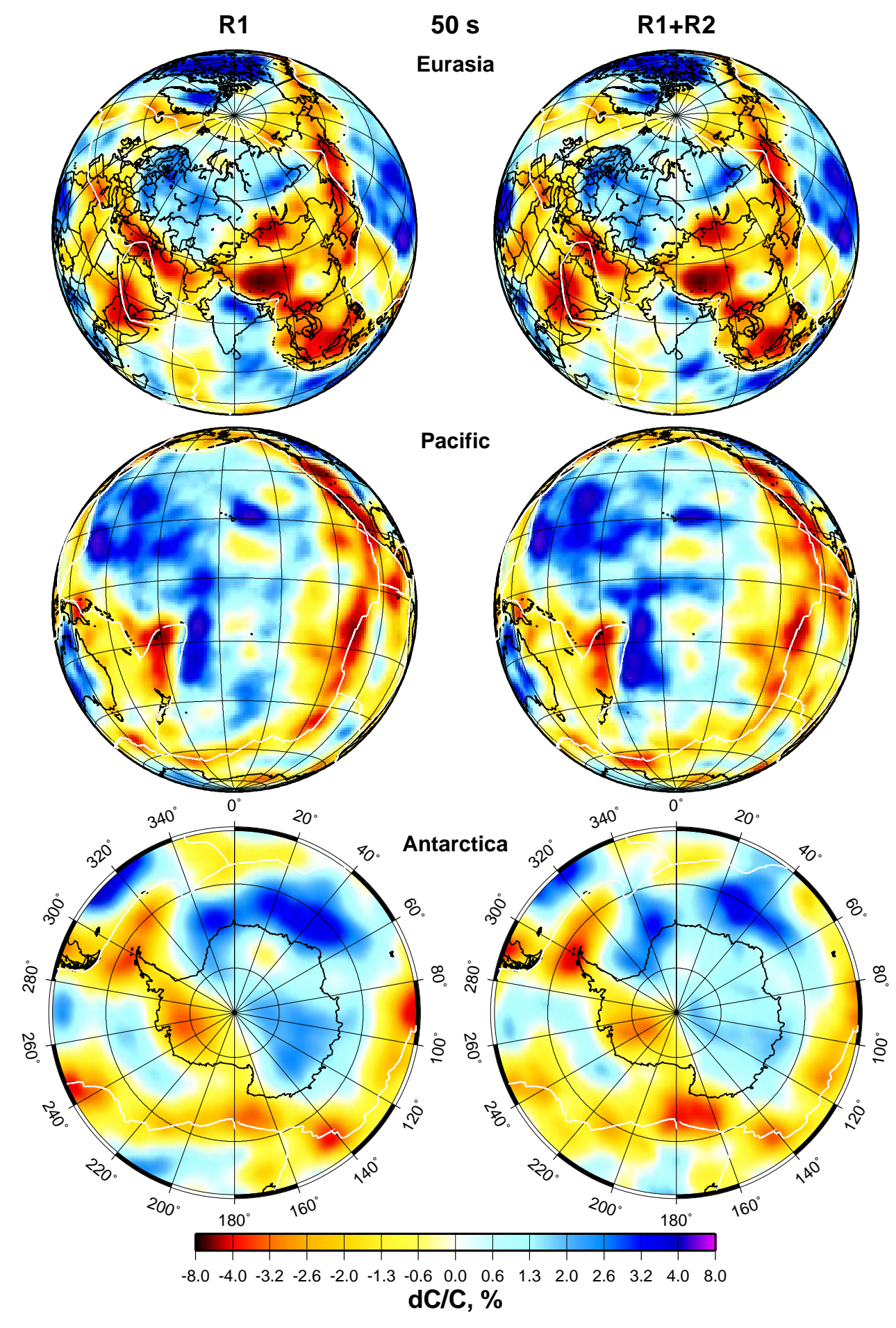

Figure 11: Tomographic maps for $50 \mathrm{sec}$ Rayleigh wave phase speeds: (LEFT) minor-arc data alone, (RIGHT) minor-arc and major-arc data combined. Results are based on the F7 sensitivity kernels (Fig. 1a). 


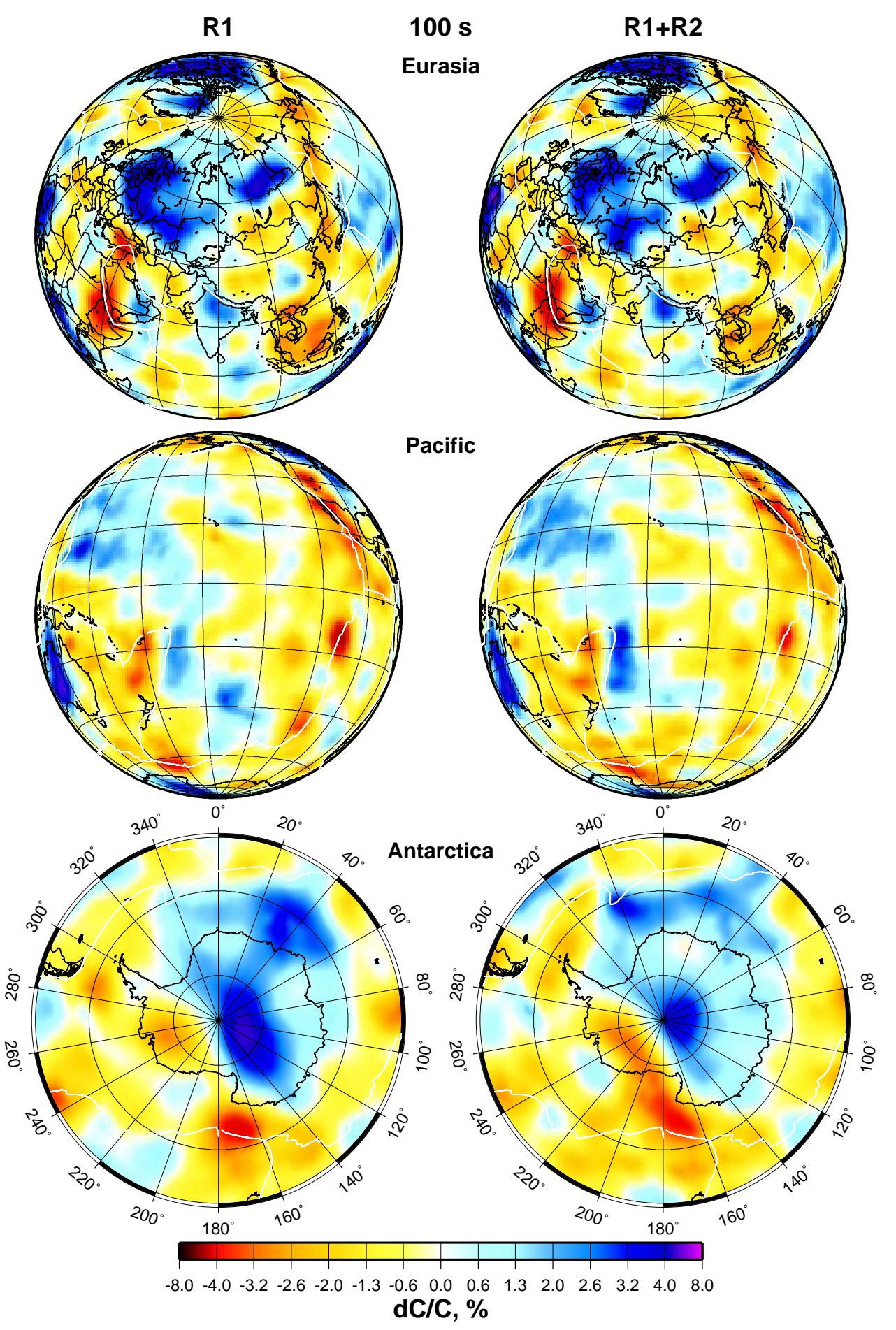

Figure 12: Same as Figure 11, but for the 100 sec Rayleigh wave phase speeds. 


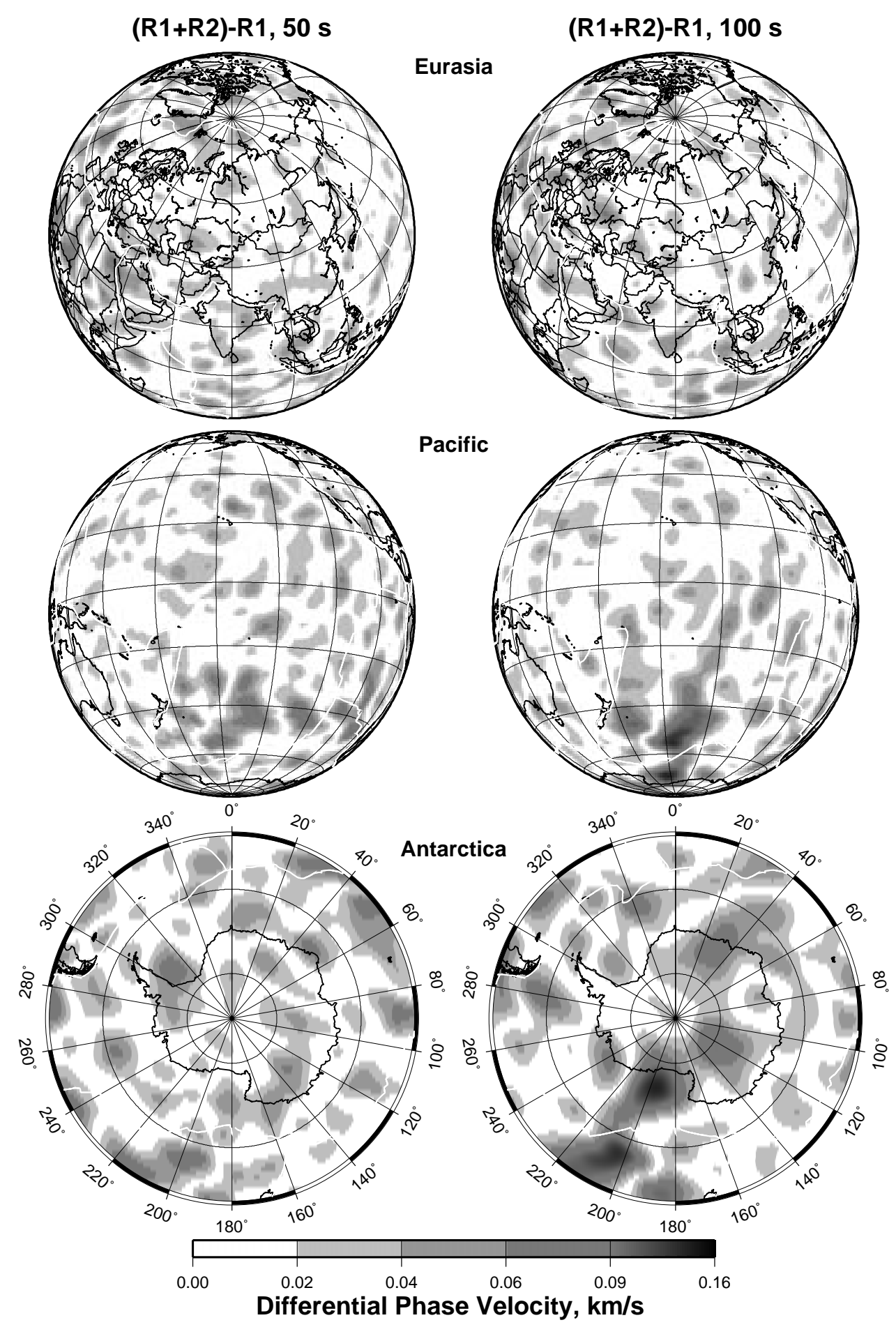

Figure 13: Absolute value of the difference between the phase speed maps constructed with both minor-arc and major-arc data and those constructed with minor-arc data alone: (LEFT) $50 \mathrm{sec}$ Rayleigh wave phase speeds, (RIGHT) $100 \mathrm{sec}$ Rayleigh wave phase speeds. Results are based on the F7 sensitivity kernels (Fig. 1a). 


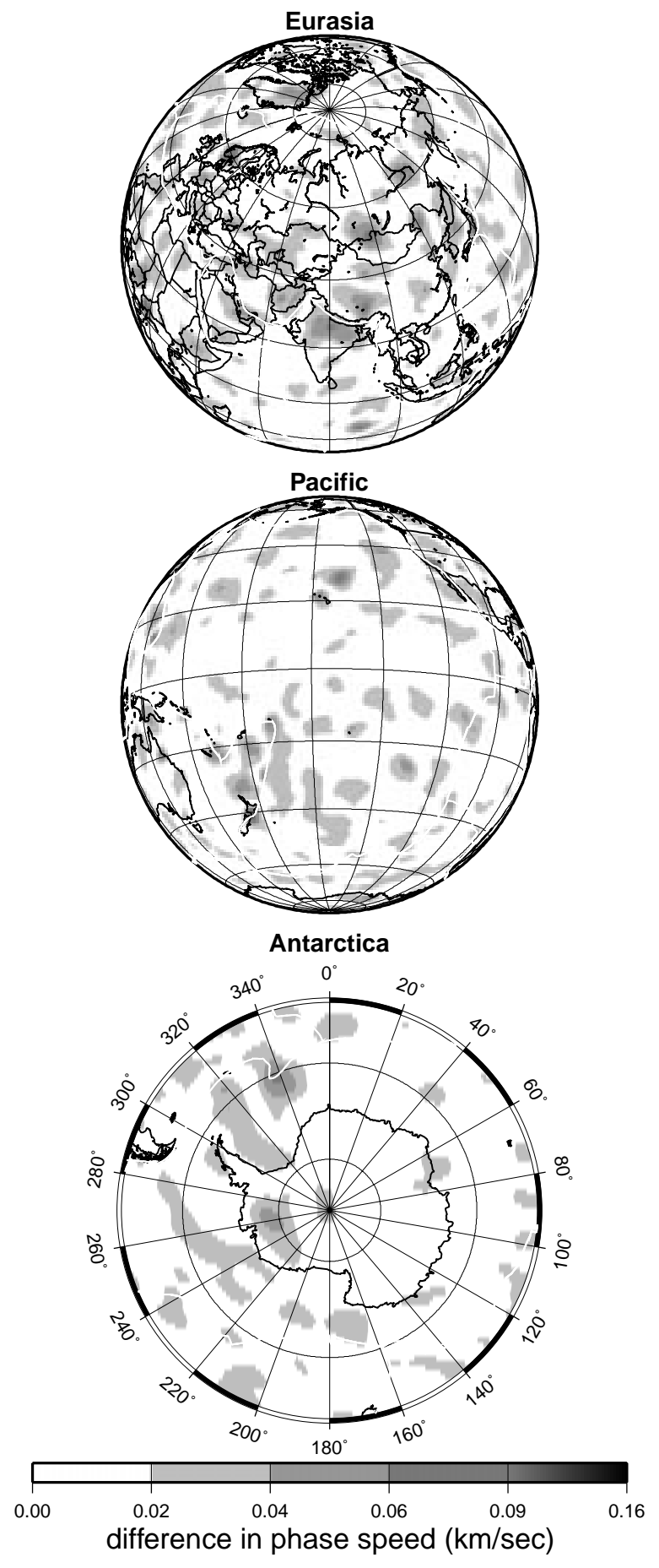

Figure 14: Absolute value of the difference between the 50 sec phase speed maps constructed with both minor-arc and major-arc data using the theory F1 (Fig. 1c) and the theory F7 (Fig. 1a). The rms of the difference is about $18 \mathrm{~m} / \mathrm{sec}(<0.5 \%)$. 


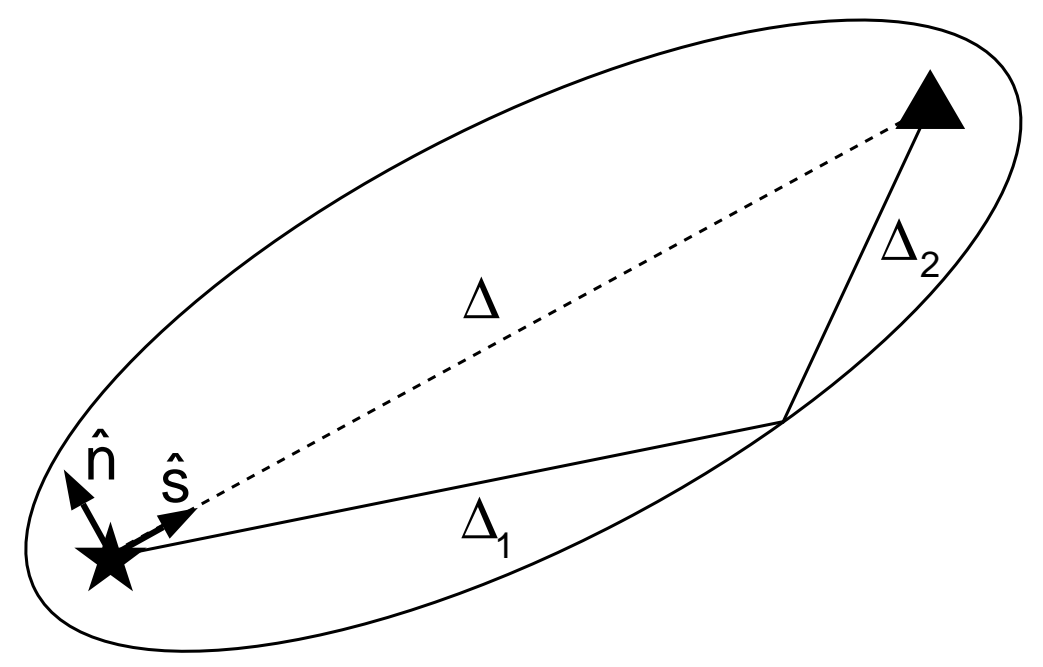

Figure 15: The first Fresnel-zone is an ellipse on a a sphere with the source (star) and receiver (triangle) at the two foci. 


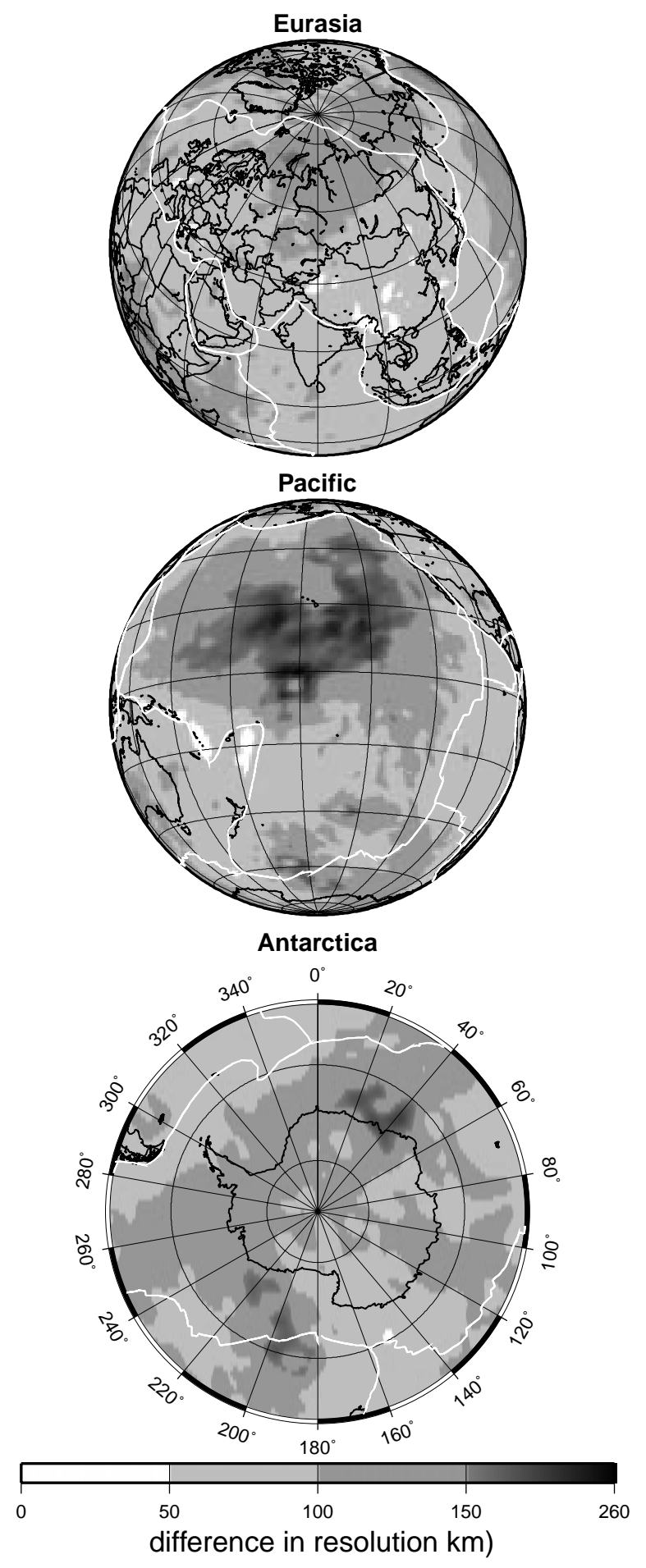

Figure 16: Difference in resolution between tomography performed with theory F1 (Fig. 1c) and theory F7 (Fig. 1a) for the $50 \mathrm{sec}$ Rayleigh wave phase speed map. Due to destructive interference among the side-lobes and the central-lobe, the wider sensitivity kernel, F7, exhibits a better resolution than the narrower kernel, F1, everywhere on the globe. 